\title{
KINEMATIC DESIGN OPTIMIZATION OF PLANER-LINK MECHANISM BASED MANIPULATOR
}

\author{
Jagdish M Prajapati \\ Department of Mechanical Engineering \\ The M S University of Baroda \\ Vadodara, India \\ j.m.prajapati-med@msubaroda.ac.in
}

\begin{abstract}
This paper presents optimized kinematic design of a planer mechanism (4-link) based planar manipulator is presented in this paper. In case of the parallel manipulator, there is only one location defined between force and motion where local mobility index is one. In this paper, for maximum local mobility index, optimum link lengths of the manipulator that is function of the location of the input link are obtained. Charts, showing the optimum kinematic design of the 4 - link planer manipulator are obtained. It is clear from this result, that the performance of the manipulator is maximum for a position interval in addition to a certain position. Also, at some positions better relationship between force and motion is observed where local mobility index is not exactly unit.
\end{abstract}

Keywords: Optimal kinematic design; Local mobility index; Force manipulability

\section{INTRODUCTION}

To design a kinematic optimum manipulator is the central focus of researchers. Some criteria are there to design robotic manipulators. Yoshikawa [1] introduced the concept of end-effector manipulability as a measure of the kinematic transmission characteristics of manipulator. One of the most important criteria in the optimal robot design is that the robot can achieve isotropic configurations. The equal forces may be exerted in all directions. Salisbury and Craig [3] proposed to use the ratio of the largest and smallest singular values of Jacobian matrix. The most manipulability measures have been derived from the condition number of the Jacobian matrix, and have been used by Asada, Chiu and Park [4], [5] and [6] for analyzing and optimizing kinematic performance of the manipulators based on the motion and force manipulability concepts. Klein and Blaho [7] use the minimum singular value as a measure. Gosselin and
Angeles [8] applied a global conditioning index to four different cases, which is based on the distribution of the condition number of the Jacobian matrix over the entire manipulator workspace. Park and Brockett [9] introduced the kinematic distortion index, which is a measure of the distortion associated with the mapping from configuration to work space. Singh and Rastegar [10] developed global velocity ellipsoid concept, which represents the velocity transmission characteristics of a manipulator. Doel and Pai [11]

introduced formalism for the systematic construction of performance measures of robot manipulators in a unified framework based on differential geometry. Kircanski [12] determined the isotropic configurations of planar and spatial manipulators in the form of polynominal. Zanganeh and Angeles [13] introduced a set of conditions for the submatrices of the Jacobians under which a parallel manipulator can attain an isotropic configuration. Matone and Roth [14] investigated the effects of actuation schemes on the minimum singular value, the manipulability and the condition number measures of kinematic performance. Bhavesh Patel [15] represented the state of art of the kinematics of planer mechanisms. Also, Bhavesh Patel [16] investigated the force effects on the planer mechanisns.

This paper presents how the link lengths are optimized for the closed-loop, four-bar planer mechanism as a manipulator with the help of local mobility index. The performance index is defined as the ratio between the minimum and maximum joint torque vector norms. For the investigated manipulator, optimum link measurements that maximize the performance index have been achieved based on the position of the input link. Comparing these results with those of the parallel manipulator, it is seen that better conditions are reached in terms of force-motion relationship with the planar manipulator. 
Nomenclature

$l_{2}$

$L_{2}$

$l_{\mathrm{a}}$

$L_{a}$

$l_{4}$

$L_{4}$

$l_{1}$

$\omega$

[j]

$\left[J_{g}\right]$

$C_{1}, C_{2}, C_{2}$

$\{\theta\}$

$\theta_{1}$

$\delta$

$\theta_{2}$

effector)

$\in$

$\left\{\frac{\pi}{r}\right\}$

$\mu$

$\left\{P_{\}}\right\}$ length of the input link 2

length ratio of the input link 2

length of the coupler link

length ratio of the coupler link

length of the output link

length ratio of the output link

length of the input link 1

derivative of $9_{2}$ with respect to $q$

Jacobian matrix

generalized Jacobian matrix

coefficients of Freudenstein's equation

vector of joints rates

rotation of the input link 1

rotation of the output link

rotation of the output link (end-

end-effector link length ratio

vector of cartesian velocities

local mobility index

rotation of the input link 2

\section{MOTION MANIPULABILITY CHARACTERISTICS}

The planar manipulator with a four-bar mechanism under study is shown in Fig. 1. Planar manipulator with a four-bar mechanism has two actuators that are fixed to the base link and drive the two input links. One of the links in a four-bar mechanism connected to the input link 1 oscillates while the other has a full rotation. Manipulator with a parallelogram mechanism is a special case of the four-bar mechanism consisting of equal two opposite links.

When the end-effector position is taken as the task domain position vector, the Jacobian matrix is defined as the matrix representing the transformation mapping the joint rates into the cartesian velocities. This transformation is written as

$$
[J]\{\theta\}=\{\ddot{p}\}
$$

Where $\{\theta\}=\left\{\begin{array}{ll}\theta_{1} & \dot{p}\end{array}\right\}^{T}$ is the vector of joints rates and $\{\dot{r}\}$ is the vector of cartesian velocities, the Jacobian matrix is given by

$$
[j]=\left[\begin{array}{ll}
-I_{1} \sin \theta_{1}-I_{1} E \sin \left(\theta_{1}+\theta_{2}\right) & -I_{1} E \sin \left(\theta_{1}+\theta_{2}\right) \omega \\
I_{2} \cos \theta_{2}+I_{2} E \cos \left(\theta_{2}+\theta_{2}\right) & \mathbb{I}_{2} E \cos \left(\theta_{2}+\theta_{2}\right) \omega
\end{array}\right]
$$

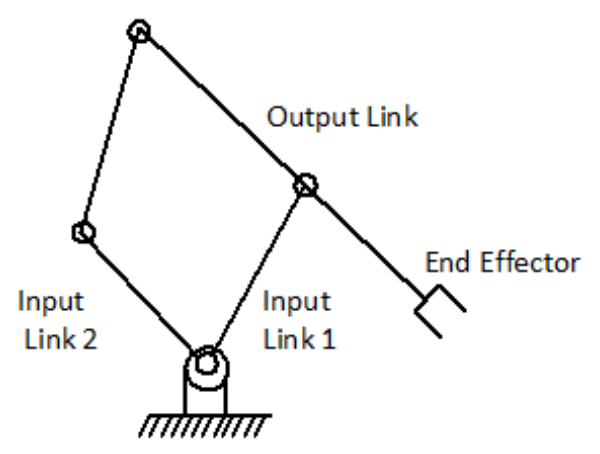

Fig. 1. The planar manipulator with a four-bar mechanism.

where $\omega=d \theta_{2} / d p$ is the derivative of the auxiliary coordinates $\theta_{2}$ with respect to $p$ and $\epsilon$ is the link length ratio. As seen in Fig. $1, \theta_{2}=\delta+\pi$ expression can be written. In this case, $\omega=d \theta_{2} / d p=d \delta / d p$ is obtained. The derivative $\omega$ can be determined by Freudenstein's equation. Freudenstein's equation is a displacement equation for the four-bar linkage, which holds true for each position of the linkage. In a compact form, it writes

$$
x \sin \delta+y \cos \delta+z=0
$$

Where $\quad x=\sin p, \quad y=C_{2}+\cos p$, $z=C_{1} \cos p+C_{\mathrm{a}}, \quad C_{1}=1 / L_{4}$,

$$
\begin{array}{cc}
C_{2}=-1 / L_{2}, & C_{\mathrm{a}}=\left(L_{\mathrm{a}}{ }^{2}-L_{2}{ }^{2}-L_{4}{ }^{2}-1\right) / 2 L_{2} L_{4}, \\
L_{2}=l_{2} / l_{1}, & \\
& L_{a}=l_{\mathrm{a}} / l_{1}, L_{4}=l_{4} / l_{1}
\end{array}
$$

$$
\sin \delta=\frac{2 \tan (\theta / 2)}{1+\tan ^{2}(6 / 2)}, \quad \cos \delta=\frac{1-\tan ^{2}(\theta / 2)}{1+\tan ^{2}(6 / 2)}
$$

The angle $\delta$ is found explicitly as a function of $p$ and the parameters $L_{2}, L_{3}, L_{4}$. Such a solution is obtained by expressing $\sin \delta$ and $\cos \delta$ in terms of $\tan (\delta / 2)$, 
(6)

$$
\delta=2 \arctan \left(\frac{x-\sqrt{x^{2}+y^{2}-z^{2}}}{y-z}\right)
$$

$$
\theta_{2}=\pi+\delta
$$

and substituting those values in Eq. (3), the angle $\theta_{2}$ is found as shown below

Using the above equations, derivative $\omega$ in the Jacobian matrix is expressed as

$$
\omega=\frac{d \delta}{d \rho}=-\frac{d(\cos a)}{d \rho}\left(\frac{1}{\sin a}\right)
$$

$$
\begin{gathered}
\text { or } \\
\omega=\frac{d \delta}{d \rho}=-\frac{d(\cos \delta)}{d \rho}\left(\frac{1}{\cos \theta}\right)
\end{gathered}
$$

The generalized Jacobian matrix, $\left[J_{g}\right]$, defined as the quadratic form of the Jacobian matrix, $[j]$, is used to characterize the force manipulability of the mechanism. $\left[b_{g}\right]$ can be written as the product of two matrices:

$$
\left[J_{g}\right]=[j][j]^{T}
$$

The propagation from the input joint torques to the output end effector force is directly proportional to the eigenvalues of $\left[J_{g}\right]$. If the eigenvalues of the generalized Jacobian matrix are $\sigma_{\max }$ and $\sigma_{\min }$, one can conclude that when the endpoint force is in the direction of eigenvector $e_{\max }$ related to the maximum eigenvalue of []$\left._{g}\right]$, the largest to exert a unit endpoint force.

Thus, the force manipulability at the mechanism's endpoint is at its worst. On the other hand, the force manipulability is at its best when a force is exerted in the direction of eigenvector $\mathrm{e}_{\min }$. The local mobility index (LMI) is denned as the ratio between the minimum and maximum eigenvalues of the generalized Jacobian matrix $\left[J_{g}\right]$, Lee (1993):
The local mobility index is bounded as $0 \leq L M I \leq 1$. The best-conditioned point is identical to an isotropic point where local mobility index equals one. This indicates that the joint torque vector norms are equal for endpoint force exerted in any direction. The isotropic point is a special condition where the mechanism has a uniform mobility in all motion directions.

The local mobility index of the planar manipulator shown in Fig. 1 can be derived from the generalized Jacobian matrix $\left[J_{g}\right]$ as

$$
\begin{aligned}
& L M I=
\end{aligned}
$$

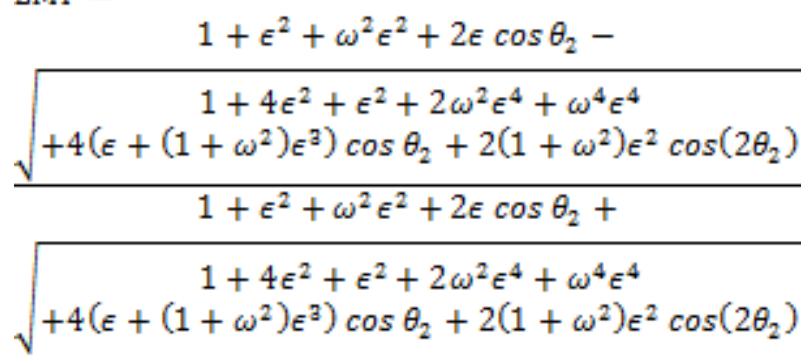

The expression LMI in Eq. (12) defines a fivevariable function depending on the input link position $\rho$ as seen in Eqs. (4)-(9) and dimensionless link lengths $L_{2}, L_{3}, L_{4}$ and $\epsilon$. For the subject of optimal kinematic design of planar manipulator with four-bar mechanism, variables $L_{2}, L_{3}, L_{4}$, which are the characteristic dimensions of this mechanism and variable $E$, which determines the position of the end-effector on output link have been used. In order to generalize the results, using the length of input link 1, link lengths have been made dimensionless. The design variables $\epsilon, L_{2}$ and $L_{4}$ which maximize the objective function LMI have been searched in the study. In the derived numerical results, first $\epsilon$ and later $\epsilon, L_{2} ; \epsilon ., L_{2}$ and $L_{4}$ variables left free, respectively and the optimization problem is solved giving constant values to the other variables. The unconstrained optimal design problem is solved by employing Newton's method iteratively, Papalambros (2000).

\section{NUMERICAL EXAMPLES}

The performance index of the manipulator for a parallel planar manipulator with equal opposite link lengths is drawn in Fig. 2 using $L_{2}=0.3, L_{3}=1$ and $L_{4}$ $=0.3$ numerical values and depending on input link and 
end-effector position. As seen in this figure, the $\rho=\pi / 4$ position of the input link and $\varepsilon=0.707107$ value for the end-effector position equal local mobility index to one in such a parallel manipulator. These values obtained for the manipulator with parallelogram mechanism are the same with results for the planar two link manipulator obtained in the studies of optimal kinematic design by Gosselin and Angeles [8], Singh and Rastegar (1995), Kircanski (1996) and Lee et al. (1993).

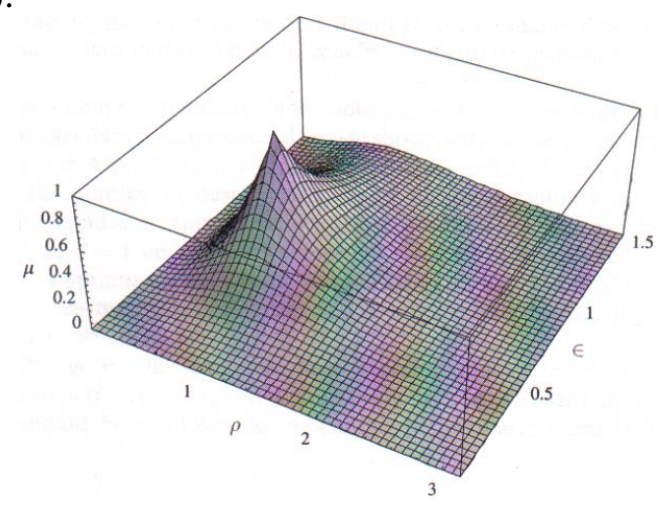

Fig. 2. The local mobility index $\mu$ versus joint angle $\rho$ and the link length ratio $\equiv$ for parallel manipulator.

Fig. 3 displays the maximum local mobility index values obtained by the optimization of different link lengths of the manipulator depending on the position $\rho$. As seen in $\mu_{\varepsilon}$ graph (full line), if only the $E$ value is optimized, local mobility index equals 1 at $\rho=\pi / 4, E=$ 0.707107 point. With $L_{3}=1, L_{4}=0.3$ constant values and $\epsilon$ and $L_{2}$ as design variables graph $\mu_{E L 2}$ (dotted line), with $L_{3}=1$ and $\epsilon, L_{2}$ and $L_{4}$ as

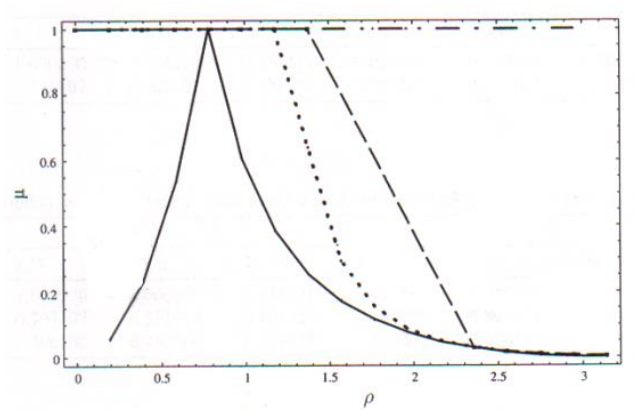

Fig. 3. The maximum local mobility index $\mu \mathrm{v} / \mathrm{s}$ joint angle $\rho\left(\mu_{x}, L_{2}=0.3, L_{3}=1, L_{4}=0.3\right)$ design variables graph $\mu_{\text {eLILA }}$ (dashed line) are derived. It is seen in these graphs that with the variable-length manipulator, the performance index can be kept at its maximum value in a position interval. As the number of free design variable increase, force-motion quality rises. As seen in the $\mu_{E L 2}$ graph, it is possible to keep performance index constant at its maximum value from $\rho=0$ to approximately $1.2 \mathrm{rad}$. position by the optimization of $l_{2}$ and $E$ variables. This position can be increased to approximately $1.4 \mathrm{rad}$. by the optimization of $\epsilon, L_{2}$ and $L_{4}$. Such a manipulator which has a higher kinematic performance than that of a parallel manipulator can be realized through hydraulically actuated linkage.

\section{CONCLUSION}

Optimal kinematic design of planar manipulator with a four-bar mechanism has been presented. The performance index is defined as the ratio called local mobility index between the minimum and maximum eigenvalues of the generalized Jacobian matrix. For the manipulator, the link measurements that maximize the performance index depending on the input link position have been found. The attained results have been compared to those of the parallel manipulator and it is seen that better results have been achieved in terms of force-motion relationship. The force-motion performance of the manipulator can be elevated by the optimization of two design variables with setting the input link long and end-effector position short formerly, from the beginning of the motion and vice versa latter. In this case, the performance index can be kept at its highest value from $\rho=0$ to approximately $1.2 \mathrm{rad}$. position. If the optimization problem is solved with three variables as the output link length is added among the design variables, it is possible to raise this interval to $1.4 \mathrm{rad}$. As a result of the comparisons, better conditions have been obtained in terms of forcemotion relationship also at the other points in addition to this maximum value of the performance index. As the number of free design variables increases, quality of the force-motion is possible to be raised. 
ELK

Asia Pacific Journals

\section{REFERENCES}

[1]. T. Yoshikawa, (1985) Dynamic manipulability of robot manipulators, Journal of Robotics Systems 2 (1) pp. 113-124.

[2]. T. Yoshikawa, (1985) Manipulability of robotic mechanism, The International Journal of Robotics Research 4 (2) pp. 3-9.

[3]. J.K. Salisbury, J.J. Craig, (1982) Articulated hands: force control and kinematic issues, International Journal of Robot Research 1 (1) pp. 4-17.

[4]. H. Asada, K. Yosef-Toumi, (1986) Analysis and design of a direct-drive arm with a five-bar-link parallel drive mechanism, ASME Journal of Dynamic Systems Measurement and Control 106, pp. 225-230.

[5]. S.L. Chiu, (1988) Task compatibility of manipulator postures, The International Journal of Robotics Research 75(5), pp. 13-21.

[6]. F.C. Park, J.W. Kim, (1988) Manipulability of closed chains, ASME Journal of Mechanical Design 120, pp. 542-548.

[7]. C.A. Klein, B.E. Blaho, (1987) Dexterity measures for the design and control of kinematically redundant manipulators, The International Journal of Robotics Research 6 (2), pp. 72-83.

[8]. C. Gosselin, J. Angeles, (1991) A global performance index for the kinematic optimization of robotic manipulators, Journal of Mechanical Design 113, pp. 220-226.

[9]. F.C. Park, R.W. Brockeet, (1994) Kinematic dexterity of robotic mechanisms, The International Journal of Robotics Research 13 (1), pp. 1-15.

[10]. J.R. Smigh, J. Rastegar, (1995) Optimal synthesis of robot manipulators based on global kinematic parameters, Mechanism and Machine Theory 30 (4), pp. 569-580.

[11]. J. K. Doel, D. Pai, (1996) Performance measures for robot manipulators: a unified approach, The International Journal of Robotics Research 15 (1), pp. 92-111.

[12]. M. Kircanski, (1996) Kinematic isotropy and optimal kinematic design of planar manipulators and a 3-DOF spatial manipulator, The International Journal of Robotics Research 15 (1), pp. 61-77.

[13]. K.E. Zanganeh, J. Angeles, (1997) Kinematic isotropy and the optimum design of parallel manipulators, The International Journal of Robotics
ELK Asia Pacific Journals - Special Issue

ISBN: 978-81-930411-4-7

Research 16 (2), pp. 185-197.

[14]. R. Matone, B. Roth, (1997) The effects of actuation schemes on the kinematic performance of manipulators, Journal of Mechanical Design 119, pp. 212-217.

[15]. B. P. Patel, J. M. Prajapati, (2011) A review on Kinematics of Hydraulic Excavator's Backhoe Attachment, International journal of engineering science and technology, 3(3), pp. 1990 - 1997.

[16]. B. P. Patel, J. M. Prajapati, (2012) Evaluation of Resistive Force using Principle of Soil Mechanics for Mini Hydraulic Backhoe Excavator, International Journal of Machine Learning and Computing, Vo. 2 No. 4, pp. $386-391$. 